\title{
The exoproteomes of clonally related Staphylococcus aureus strains are diverse
}

\begin{abstract}
Several studies have shown that protein expression patterns vary in unrelated bacterial strains due to genomic plasticity and gene regulation, resulting in enhanced heterogeneity in the infection potential. However, exoprotein expression patterns of closely related clonal strains have not been well characterized. Here, we used medium-range $(\mathrm{pH} 4-7)$ immobilized $\mathrm{pH}$ gradient-two-dimensional gel electrophoresis to investigate the exoproteome from closely related Staphylococcus aureus clonal isolates. Interestingly, we found that, under identical in vitro experimental conditions, a number of protein spots were uniquely present in samples from each clonal isolate irregardless of the similarity of the genotype and the same virulence gene profile. Only a few abundant invariant proteins were found among identical genotypic isolates. Our results clearly shown that heterogeneity in the exoproteome was present even among clonally related strains. We suggest that this heterogeneity may contribute to the degree of virulence even within one clonal genotype. The heterogeneity in the exoproteome of closely related S. aureus strains observed in the current study postulates that pre-existing antibodies are not very protective during recurrent infection with the same strain. Therefore, our findings underscore the importance of taking all clonally related strains into account during proteome analyses.
\end{abstract}

Keyword: Staphylococcus aureus; Exoprotein; 2-DGE; Closely related clonal 